\title{
Short fractionation radiotherapy for early prostate cancer in the time of COVID-19: long-term excellent outcomes from a multicenter Italian trial suggest a larger adoption in clinical practice
}

\author{
Salvina Barra ${ }^{1}$ - Alessia Guarnieri ${ }^{2} \cdot$ Michela Buglione di Monale e Bastia $^{3} \cdot$ Michela Marcenaro $^{1}$ - Elena Tornari ${ }^{4}$. \\ Liliana Belgioia ${ }^{1,4}$ (1) $\cdot$ Stefano Maria Magrini ${ }^{3} \cdot$ Umberto Ricardi $^{2} \cdot$ Renzo Corvò $^{1,4}$
}

Received: 25 March 2020 / Accepted: 27 April 2020 / Published online: 15 May 2020

(c) Italian Society of Medical Radiology 2020

\begin{abstract}
Introduction To evaluate stereotactic body radiotherapy (SBRT) in low-risk Prostate Cancer patients as preferred treatment option in emergency health conditions.

Materials and methods From April 2013 to September 2015, 28 patients with low-risk prostate cancer were prospectively enrolled. The SBRT prescribed dose was 36.25 Gy in 5 fractions, twice a week. Primary endpoints were acute and late toxicity. Secondary endpoints were biochemical recurrence free survival (bRFS) and overall survival.

Results Median follow-up was 65.5 months (range 52-81). No acute G3 or G4 toxicity was recorded. Acute G1 or G2 genitourinary (GU) toxicity occurred in $43 \%$ and acute G1-G2 gastrointestinal (GI) toxicity in 14\%. Late G1 and G3 GU toxicity in $18 \%$ and $3.5 \%$, respectively. The G3 toxicity was not directly attributable to radiotherapy. Late G1 GI toxicity occurred in $18 \%$. 5yy bRFS was $96.5 \%$ (95\% CI $82.3-99.4 \%)$.

Conclusions Stereotactic body radiotherapy for early prostate cancer reported safe toxicity profile and a good clinical outcome at the median follow-up of 5 years. It may be an useful option if radiotherapy is required in emergency medical conditions.
\end{abstract}

Keywords COVID-19 $\cdot$ SBRT $\cdot$ Prostate cancer $\cdot$ Hi-tech $\cdot$ Hypofractionated radiotherapy

\section{Introduction}

The rapid development of Coronavirus Disease 2019 (COVID-19) in the whole world led the WHO to declare on $11^{\mathrm{Th}}$ March 2020 a pandemic. In this situation, radiation oncologist are called to optimize the management of cancer patients that often are frail and cannot afford to postpone treatment [1]. Some preliminary suggestions have been indicated, but there is still no clear consensus [2, 3]. However,

Liliana Belgioia

liliana.belgioia@unige.it

1 Radiation Oncology Department, IRCCS Ospedale Policlinico San Martino, Genoa, Italy

2 Radiation Oncology Department AO Città della Salute e della Scienza - Molinette, University of Turin, Turin, Italy

3 Radiation Oncology Department, University and Spedali Civili of Brescia, Brescia, Italy

4 Health Science Department (DISSAL), University of Genoa, Genoa, Italy the use of radiotherapic hypofractionation for some cancers seems to be shared worldwide.

Prostate cancer (PCa) is the second most common cancer in men with an estimated number of new diagnosis of 164.700 in USA and 450.000 in Europe for 2018 [4, 5] and the majority of cases being diagnosed at early stage of disease [6]. Several treatments are available, and external beam radiotherapy (EBRT) is one of the first line options [7, 8].

In version 1 of 2020 NCCN Guidelines for Prostate Cancer, moderate hypofractionation regimens are indicated as preferred and several literature data demonstrate that, they are not inferior to standard fractionation (i.e. $74-81 \mathrm{~Gy}$ in 37-45 fractions) [9]. So, several schedules on 20-28 fractions (dose per fraction: 2.75-3 Gy) are widely adopted [10]. However, in the COVID-19 pandemic context, 20 fractions seem also demanding for obvious reasons not only for patients but also for the radiation department. A possible alternative is ultra-hypofractionated courses (6.1-8 Gy per fraction up to 5-7 fractions) that, to date, are considered an appropriate regimen option across all risk groups of patients (from very low to high and very high risk) (NCCN 2020, v1) 
[9]. It is well known that, in prostate cancer, radiation dose intensification correlates with better oncological outcomes and SBRT has shown acceptable efficacy and toxicity and has become "a" standard of care [11]. Despite the scientific evidence, SBRT has been exported and used only in large centers or in academic institutions but not taken into consideration in other radiotherapy centers although they have Hi-Tech accelerators [12]. The main concern is related to the risk of inducing severe late toxicity in patients with a good prognosis [13]. Here, we report the long-term results of a phase II multicenter prospective trial on Stereotactic Body Radiotherapy in low-risk prostate cancer patients. In this paper, we suggest that SBRT can be cost-effective with minimal toxicity and can therefore be offered more to patients, especially in emergency health conditions.

\section{Materials and methods}

The study was approved by the research Ethics Board (N. RITS. 13.001), registered on clinicaltrials.gov (NCT 02423889) and conducted in accordance with the Declaration of Helsinki and Good Clinical Practice guidelines. Written, informed consent was obtained from all participants.

From April 2013 to September 2015, 28 of 30 planned patients were enrolled $(17-61 \%, 5-18 \%$ and $6-21 \%$ at IRCCS Ospedale Policlinico San Martino in Genoa, ASST Spedali Civili in Brescia and A.O.U. Città della Salute e della Scienza in Turin, respectively).

\section{Patient selection and treatment details}

The eligibility criteria for enrollment were the following: histologically confirmed prostate adenocarcinoma, age $>18$ years, life expectancy $>10$ years, low-risk group (according to $2013 \mathrm{NCCN}$ classification), PSA $\leq 10 \mathrm{ng} / \mathrm{mL}$, cT1-T2a, no nodal involvement, no distant metastasis, no concomitant ADT, no previous prostatectomy, good performance status $(\mathrm{ECOG}<2)$. Exclusion criteria were: intermediate, high, very high or advanced disease, nodal involvement, distant metastasis, presence of inflammatory intestinal and autoimmune diseases in the acute phase, anticoagulant therapy in progress, hip replacement or other abdominalpelvic devices that make it impossible to acquire the images necessary for planning, previous pathology malignant neoplastic excluded basal cell skin cancer, previous pelvic radiotherapy and mental disease that cannot ensure a valid informed consent.

Patients were staged with an accurate anamnesis, clinical examination, rectal digital exploration (DRE), initial PSA and routine blood exams, pelvic multi-parametric magnetic resonance imaging (mp-MRI), chest computer tomography (CT), bone scan (although both chest $\mathrm{CT}$ and bone scan would not be required by NCCN guidelines), prostate biopsy with at least 6 fragments. Rectoscopy was performed only in case of suspected inflammatory diseases or intestinal diverticulosis.

A treatment planning CT scan was carried out from L1 to the proximal third of the femurs with $2.5 \mathrm{~mm}$ slices.

All patients were immobilized in the supine position with the arms on the chest using dedicated immobilization systems with a comfortable full bladder and empty rectum. Patients were instructed to drink $500 \mathrm{~mL}$ of water $30 \mathrm{~min}$ before CT scan and treatment. The SBRT prescribed dose was 36.25 Gy in 5 fractions, twice a week with a Biologically Effective Dose (BED) of $211 \mathrm{~Gy}$. The clinical target volume (CTV) included the prostate, and a $5 \mathrm{~mm}$ margin was added to CTV to obtain the planning target volume (PTV). The CTV was countered using a deformable registration of T2 MRI sequences with the planning CT. Organs at risk (OARs) constraints were the following: V36 Gy $<1$ cc, V100<1.8 Gy, V90<3.6 Gy, V80<7.3 Gy, $\mathrm{V} 50<18.25$ Gy for rectum, V36Gy $\leq 1 \mathrm{cc}, \mathrm{V} 37 \leq 1 \mathrm{cc}$, $\mathrm{V} 50 \leq 40 \%$, V $100 \leq 10 \%$ for bladder, V $29.5 \mathrm{~Gy}<50 \%$ for penile bulb, V $20 \leq 54 \%$ for femoral heads and $\leq 10 \%$ of prescription dose for small and large bowel outside the PTV.

SBRT was delivered by Helical Tomotherapy Hi.Art (Genoa and Brescia) and Elekta Axesse (Turin) using VMAT with an integrated system of Image-Guided Radiotherapy (IGRT). Daily IGRT setup verification and correction with Megavoltage CT (MVCT) with Tomotherapy or Cone Beam CT (CBCT) with Axesse was performed before each fraction. Planning optimal goals included for the PTV $\mathrm{V} 95 \%>98 \%, \mathrm{~V} 103 \%<3 \%$ and $\mathrm{V} 105 \%<0.5 \mathrm{cc}$ and for the CTV V95\%> $99 \%$.

\section{Follow-up and toxicity assessment}

Patients were assessed with clinical evaluations and PSA at 1-month after RT, then every 3 months for the first year, then twice a year for the following four years and annually thereafter. The acute and late toxicity according to the CTCAE version 4.0 and RTOG/EORTC scale was recorded. The biochemical relapse was defined according to the American Society for Therapeutic Radiology and Oncology (ASTRO) definition (nadir $+2 \mathrm{ng} / \mathrm{mL}$ ).

\section{Endpoints}

Primary endpoints were acute and late toxicity. Secondary endpoints were biochemical recurrence free survival (bRFS), and overall survival (OS). bRFS was calculated from the date of end of treatment and the date of the diagnosis of the biochemical recurrence; OS was calculated from the date of the end of treatment to the date of the patient's death due to any cause. 


\section{Statistical analysis}

The planned sample size was 30 patients. Patient accrual was expected to be completed within September 2015, at that time the accrual was stopped. Demographic and tumor characteristics were summarized using mean, median and range for continuous variables and proportions for categorical variables. The PSA nadir was set to be the lowest PSA value following treatment completion. Kaplan-Meier method was used to estimate bRFS and OS and reported as probability of being event-free at 5 years, $95 \%$ confidence interval $(95 \% \mathrm{CI})$. For these survival functions, patients alive and without events were censored at the time of last followup. All analyses were conducted using SPSS Statistics v. 22.02013 (IBM Corporation, New York, NY, USA).

\section{Results}

Patient's baseline characteristics are shown in Table 1. Median follow-up was 65.5 months (range 52-81). All patients have a localized low-risk PCa with Gleason Score (GS) $3+3=6 / 10$, PSA at diagnosis $\leq 10$ (median $5.84 \mathrm{ng}$ / $\mathrm{mL}$ ) and no one performed concomitant androgen-deprivation therapy (ADT). The median age of patients was 72 years old (range 55-80).

According to the 2013 NCCN classification of low-risk category, pts without pelvic lymph node involvement and distant metastasis but with cancer also extended to both lobes (T2c) were included; currently, they are classified as favorable intermediate-risk class; so, according to the latest NCCN classification, we had: $15 / 28$ patients (53\%) very low risk, $10 / 28$ patients $(36 \%)$ low risk and $3 / 28$ patients $(11 \%)$ favorable intermediate risk. When indicated, active surveillance was proposed but patients refused it.

Table 1 Patients characteristics

\begin{tabular}{ll}
\hline & Numbers of pts $(\%)$ \\
\hline Age & $72(55-80)$ \\
Median (range) & \\
T stage & $2(7 \%)$ \\
T1a & $1(3 \%)$ \\
T1b & $12(43 \%)$ \\
T1c & $10(36 \%)$ \\
T2a & 0 \\
T2b & $3(11 \%)$ \\
T2c & \\
PSA at baseline & $5.84(2.3-10)$ \\
Median (range) & \\
Gleason score & $28(100 \%)$ \\
$3+3$ & \\
\hline
\end{tabular}

\section{Toxicity}

None patient developed G3 or G4 acute toxicity, 12/28 patients $(43 \%)$ presented G1 or G2 genitourinary (GU) toxicity consisting in: an increase of frequency, urgency, dysuria that resolved within 1 month after the end of radiotherapy. In some cases, a mild anti-inflammatory therapy was administrated. Only 4/28 patients (14\%) presented acute G1-G2 gastrointestinal (GI) toxicity consisting of anal pain and diarrhea (3 G1 and $1 \mathrm{G} 2$ toxicity, respectively).

In regard to late toxicity, $5 / 28$ patients $(18 \%)$ presented G1 GU toxicity urinary urgency and nycturia and only one patient had erectile dysfunction with minimal bleeding ejaculation, which afterwards resolved spontaneously in less than 3 months. One patient (3.5\%) presented G3 GU toxicity; this patient developed a second bladder tumor, treated firstly by TURV and then by TURP for an episode of urinary retention with subsequent incontinence. In this case, probably the toxicity could not be attributed exclusively to radiation treatment. Concerning the late GI toxicity, only 5/28 (18\%) presented G1 toxicity with flatulence diarrhea.

\section{Oncological outcomes}

One patient developed a biochemical failure after 37 months from the end of SBRT. This patient, with a cT2aNOM0 disease stage and a PSA value of $8.49 \mathrm{ng} / \mathrm{mL}$ at diagnosis, was restaged with $18 \mathrm{~F}$ - Choline PET/CT, which resulted negative and started ADT. 5yy bRFS was 96.5\% (95\% CI 82.3-99.4\%) (Fig. 1). Three out of 28 patients (11\%) developed a second primary ( 2 bladder cancer and 1 pleural mesothelioma). At last follow-up, all patients were alive. None of them developed distant metastasis.

In regard to the PSA trend, the median PSA pre-RT value was $5.8 \mathrm{ng} / \mathrm{mL}$, dropped down to $2.37 \mathrm{ng} / \mathrm{mL}$ after 1 month then to $1.2 \mathrm{ng} / \mathrm{mL}$ at 3 months and below $1 \mathrm{ng} / \mathrm{mL}$ at 6 months. The median nadir PSA value was $0.25 \mathrm{ng} / \mathrm{mL}$ and was obtained after a median of 30 months from the end of the radiation treatment.

\section{Discussion}

In emergency medical situations, where the risk is that of not treating the patient with prostate cancer, delaying his therapy or initiating him with an inappropriate hormonal therapy, it is recommended to adopt radiotherapy schemes based on scientific evidence of efficacy, speed of execution and minimal toxicity [14]. Our analysis confirms that ultrahypofractionation schedule, as SBRT, in prostate cancer allows to obtain excellent result both in terms of acute and late toxicity and also in terms of oncological results. The literature data available to date have widely demonstrated 


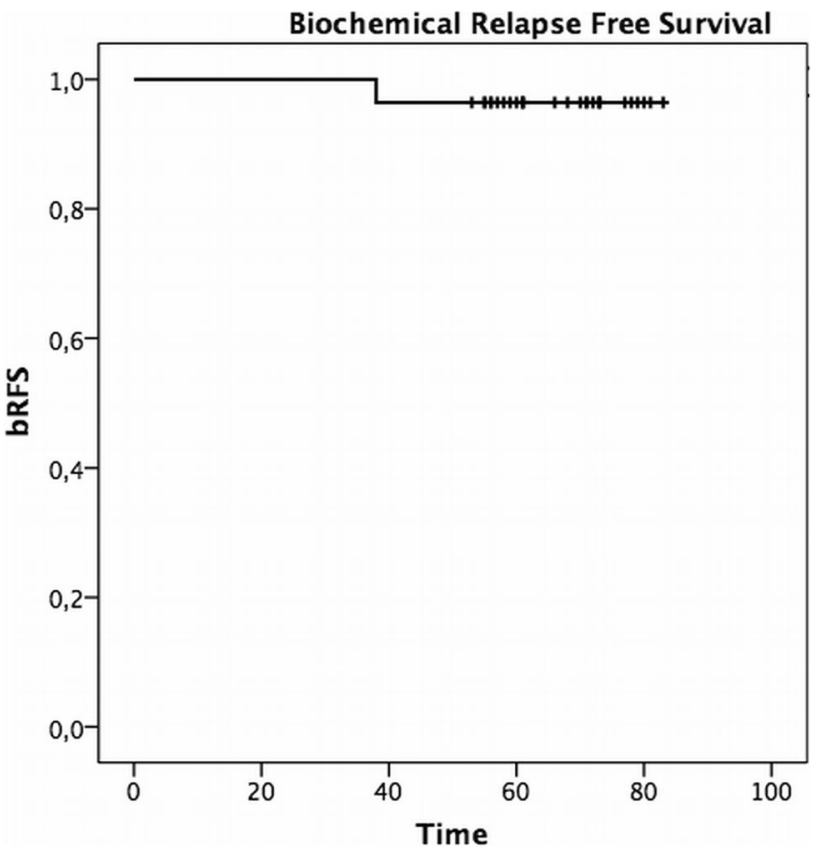

Fig. 1 Biochemical recurrence free survival

that, SBRT in these patients is not only feasible, but it is also a valid therapeutic choice and it is included as an option in any risk class groups (NCCN guidelines) [9]. A recent meta-analysis of 38 prospective studies (for a total of 6116 patients in which the intermediate risk class was the most represented group), underlined this [15]. The acute toxicity rates reported in this meta-analysis were very low with acute grade $\geq 3$ toxicity $\leq 1 \%$ (acute G3 GU toxicity in $0.5 \%$ of patients, no G4 GU toxicity and acute G3 GI toxicity in $0.06 \%$ of patients and $\mathrm{G} 4$ in $0.03 \%$ ) and late $\geq \mathrm{G} 3 \mathrm{GU}$ toxicity of $2 \%$ and late $\mathrm{G} \geq 3$ GI toxicity of $1.1 \%$ [15]. Concerning the oncological outcomes, the reported overall 5-year bRFS rate was 95.3\% (95\% CI 91.3-97.5\%), and 96.7\% and 92.1\% for the low and intermediate risk, respectively (bRFS is not available in patients at high risk because not all studies indicated division by risk classes) $[15,16]$. Even our results are completely comparable with the data reported.

In our opinion, SBRT in PCa is currently feasible in all centers equipped with hi-tech technology that includes daily IGRT, even outside a clinical trial. However, some literature data refer to patients treated with a 3DCRT technique, in particular the HYPO-RT-PC study (a randomized trial of $78 \mathrm{~Gy}$ in 39 fractions versus $42.7 \mathrm{~Gy}$ in 7 fractions in intermediate and high-risk patients) enrolled patients treated both with 3DCRT technique and with IMRT [17]. The preliminary data of the high-dose arm of RTOG 0126 trial showed a significant reduction of acute grade $\geq 2$ GI and GU toxicity in patients treated with IMRT [18], data confirmed also by a recent meta-analysis both in regards severe acute toxicity and late GI toxicity $[19,20]$.
The limitations of this analysis are mainly represented by the sample size and by the fact that there have been enrolled patients that, to date, would be classified as very-low, low and intermediate favorable risk; however, the authors stress that this is attributable to the fact that the multicenter study started in 2013 when this type of treatment did not represent a solid option, as it can be now proposed, but rather an experimental treatment.

Moreover, the authors wanted to evaluate the long-term results before adopting the scheme as routine in the selected cases with early prostate carcinoma. It might be questionable the opportunity to plan a radical treatment in some of these patients (mainly those classified as very low and low risk) when a good alternative is represented by active surveillance or in which the treatment could be postponed [14]. In our experience, however, the patients have strongly chosen an active treatment and, in daily practice, the same patients ask for stereotactic technique. Even now, the demand for radiation therapy by many males remains unchanged in the face of the desire to cure cancer.

In this moment of health emergency due to the COVID19 infection, it is clear that we are called to rethink and adapt our clinical practice [21]. Since the long-term results are satisfactory, this short fractionated radiotherapy should be strongly considered to reduce the access of patients to the radiotherapy department and subsequently reduce the risk of exposure for the patients (who are generally elderly) but also for the staff [22].

\section{Conclusions}

The indication for hypofractionation of radiation therapy is a recommendation to be adopted in emergency health conditions for many cancers including prostate cancer. At date, the evidence of excellent long-term results obtained with SBRT for prostate cancer should advise other radiation oncologists to adopt this scheme to ensure effective and safe treatment even outside clinical trials.

Author contributions All authors contributed to the study conception and design. Material preparation, data collection and analysis were performed by LB and ET. The first draft of the manuscript was written by $\mathrm{RC}, \mathrm{SB}$ and $\mathrm{LB}$, and all authors commented on previous versions of the manuscript. All authors read and approved the final manuscript.

\section{Compliance with ethical standards}

Conflict of interest The authors declare that they have no conflict of interest.

Ethics approval This study was performed in line with the principles of the Declaration of Helsinki. Approval was granted by the Ethics 
Committee of IRCCS Ospedale Policlinico San Martino, Genoa, (Date 28/01/2013/No. RITS.13.001).

Consent to participate Informed consent was obtained from all individual participants included in the study.

\section{References}

1. Achard V, Tsoutsou P, Zilli T (2020) Radiotherapy in the time of the Coronavirus pandemic: when less is better. Int J Radiat Oncol Biol Phys. https://doi.org/10.1016/j.ijrobp.2020.03.008

2. Coles CE, Aristei C, Bliss J et al (2020) International guidelines on radiation therapy for breast cancer during the COVID-19 pandemic. Clin Oncol (R Coll Radiol) 32:279-281. https://doi. org/10.1016/j.clon.2020.03.006

3. Filippi AR, Russi E, Magrini SM, Corvò R (2020) Letter from Italy: First practicalindications for radiation therapy departments during COVID-19 outbreak. Int J Radiat Oncol Biol Phys pii: S0360-3016(20)30930-5. https://doi.org/10.1016/j.ijrob p.2020.03.007

4. Siegel RL, Miller KD, Jemal A (2020) Cancer statistics, 2020. CA Cancer J Clin 70:7-30. https://doi.org/10.3322/caac.21590

5. Ferlay J, Colombet M, Soerjomataram I et al (2018) Cancer incidence and mortality patterns in Europe: estimates for 40 countries and 25 major cancers in 2018. Eur J Cancer 103:356-387. https:// doi.org/10.1016/j.ejca.2018.07.005

6. US Preventive Services Task Force, Grossman DC, Curry SJ et al (2018) Screening for prostate cancer: US Preventive Services Task Force Recommendation Statement. JAMA 319:1901-1913. https ://doi.org/10.1001/jama.2018.3710

7. Nicosia L, Mazzola R, Rigo M et al (2019) Moderate versus extreme hypofractionated radiotherapy: a toxicity comparative analysis in low- and favorable intermediate-risk prostate cancer patients. J Cancer Res Clin Oncol 145:2547-2554. https://doi. org/10.1007/s00432-019-02983-3

8. Bellavita R, Scricciolo M, Bini V et al (2016) Radiotherapy for early-stage prostate cancer in men under 70 years of age. Tumori 102:209-216. https://doi.org/10.5301/tj.5000433

9. Mohler JL, Gao X, Pow-Sang JM (2020) NCCN Guidelines Index table of contents discussion. Prostate Cancer 167:479-505

10. Barra S, Belgioia L, Marcenaro M et al (2018) Moderate hypofractionated radiotherapy after prostatectomy for cancer patients: toxicity and clinical outcome. CMAR 10:473-480. https://doi. org/10.2147/CMAR.S146131

11. Marvaso G, Ciardo D, Gandini S et al (2019) Comparison of outcomes and toxicity between extreme and moderate radiation therapy hypofractionation in localized prostate cancer: a propensity score analysis. Int J Radiat Oncol Biol Phys 105:735-744. https://doi.org/10.1016/j.ijrobp.2019.07.027

12. Buglione M, Noale M, Bruni A et al (2019) Treatment paths for localised prostate cancer in Italy: The results of a multidisciplinary, observational, prospective study (Pros-IT CNR). PLoS ONE 14:e0224151. https://doi.org/10.1371/journ al.pone.0224151

13. Pontoriero A, Iatì G, Mondello S et al (2016) High-dose robotic stereotactic body radiotherapy in the treatment of patients with prostate cancer: preliminary results in 26 patients. Technol Cancer Res Treat 15:179-185. https://doi.org/10.1177/153303461456699 4

14. Simcock R, Thomas TV, Mercy CE et al (2020) COVID-19: global radiation oncology's targeted response for pandemic preparedness. Clind Transl Radiat Oncol. https://doi.org/10.1016/j. ctro.2020.03.009

15. Jackson WC, Silva J, Hartman HE et al (2019) Stereotactic body radiation therapy for localized prostate cancer: a systematic review and meta-analysis of over 6,000 patients treated on prospective studies. Int J Radiat Oncol Biol Phys 104:778-789. https://doi. org/10.1016/j.ijrobp.2019.03.051

16. Detti B, Bonomo P, Masi L et al (2015) Stereotactic radiotherapy for isolated nodal recurrence of prostate cancer. World J Urol 33:1197-1203. https://doi.org/10.1007/s00345-014-1427-x

17. Widmark A, Gunnlaugsson A, Beckman L et al (2019) Ultrahypofractionated versus conventionally fractionated radiotherapy for prostate cancer: 5-year outcomes of the HYPO-RT-PC randomised, non-inferiority, phase 3 trial. Lancet 394:385-395. https ://doi.org/10.1016/S0140-6736(19)31131-6

18. Michalski JM, Yan Y, Watkins-Bruner D et al (2013) Preliminary toxicity analysis of 3-dimensional conformal radiation therapy versus intensity modulated radiation therapy on the high-dose arm of the Radiation Therapy Oncology Group 0126 prostate cancer trial. Int J Radiat Oncol Biol Phys 87:932-938. https://doi. org/10.1016/j.ijrobp.2013.07.041

19. Yu T, Zhang Q, Zheng T et al (2016) The effectiveness of intensity modulated radiation therapy versus three-dimensional radiation therapy in prostate cancer: a meta-analysis of the literatures. PLoS ONE 11:e0154499. https://doi.org/10.1371/journal.pone.0154499

20. De Felice F, Musio D, Caiazzo R et al (2014) Two different intensity-modulated radiotherapy strategies for patients with high-risk prostate cancer. Anticancer Res 34:3747-3751

21. Corvò R, Lamanna G, Vagge S et al (2013) Once-weekly stereotactic radiotherapy for patients with oligometastases: compliance and preliminary efficacy. Tumori 99:159-163. https://doi. org/10.1700/1283.14186

22. Brand DH, Tree AC, Ostler P et al (2019) Intensity-modulated fractionated radiotherapy versus stereotactic body radiotherapy for prostate cancer (PACE-B): acute toxicity findings from an international, randomised, open-label, phase 3, non-inferiority trial. Lancet Oncol 20:1531-1543. https://doi.org/10.1016/S1470 $-2045(19) 30569-8$

Publisher's Note Springer Nature remains neutral with regard to jurisdictional claims in published maps and institutional affiliations. 